\title{
Neurophysiology and Neuroanatomy of Pitch Perception: Auditory Cortex
}

\author{
MARK JUDE TRAMO,${ }^{a, b, c}$ PETER A. CARIANI, ${ }^{a, e}$ CHRISTINE K. KOH, ${ }^{a, b}$ \\ NIKOS MAKRIS,,$^{a, d}$ AND LOUIS D. BRAIDA ${ }^{b}$ \\ ${ }^{a}$ Department of Neurology, Harvard Medical School, and Massachusetts General \\ Hospital; The Institute for Music and Brain Science, Auditory Neuroscience Program, \\ Boston, Massachusetts 02114, USA \\ ${ }^{b}$ Sensory Communication Group, Research Laboratory of Electronics, \\ Massachusetts Institute of Technology, Cambridge, Massachusetts 02139, USA \\ ${ }^{c}$ Eaton-Peabody Laboratory of Auditory Physiology, \\ Massachusetts Eye and Ear Infirmary, Boston, Massachusetts 02114, USA \\ ${ }^{d}$ Center for Morphometric Analysis, Martinos Center for Biomedical Imaging, \\ Massachusetts General Hospital, Charlestown, Massachusetts 02129, USA \\ ${ }^{e}$ Department of Physiology, Tufts Medical School, Boston, Massachusetts 02111 USA
}

\begin{abstract}
We present original results and review literature from the past fifty years that address the role of primate auditory cortex in the following perceptual capacities: (1) the ability to perceive small differences between the pitches of two successive tones; (2) the ability to perceive the sign (i.e., direction) of the pitch difference [higher (+) vs. lower (-)]; and (3) the ability to abstract pitch constancy across changes in stimulus acoustics. Cortical mechanisms mediating pitch perception are discussed with respect to (1) gross and microanatomical distribution; and (2) candidate neural coding schemes. Observations by us and others suggest that (1) frequency-selective neurons in primary auditory cortex (A1) and surrounding fields play a critical role in fine-grained pitch discrimination at the perceptual level; (2) cortical mechanisms that detect pitch differences are neuroanatomically dissociable from those mediating pitch direction discrimination; (3) cortical mechanisms mediating perception of the "missing fundamental frequency (F0)" are neuroanatomically dissociable from those mediating pitch perception when Fo is present; (4) frequency-selective neurons in both right and left A1 contribute to pitch change detection and pitch direction discrimination; (5) frequency-selective neurons in right $\mathrm{A1}$ are necessary for normal pitch direction discrimination; (6) simple codes for pitch that are based on single- and multiunit firing rates of frequency-selective neurons face both a "hyperacuity problem" and a "pitch constancy problem"-that is, frequency discrimination thresholds for pitch change direction and pitch direction discrimination are much smaller than neural tuning curves predict, and firing rate patterns change dramatically under conditions in which pitch percepts remain invariant; (7) cochleotopic organization of frequency-selective neurons bears little if any relevance to perceptual acuity and pitch constancy;
\end{abstract}

Address for correspondence: Mark Jude Tramo, M.D., Ph.D., Director, The Institute for Music and Brain Science, 175 Cambridge Street, Suite 340, Boston, MA 02114. Voice: 617-726-5409. mtramo@hms.harvard.edu; http://www.brainmusic.org

Ann. N.Y. Acad. Sci. 1060: 148-174 (2005). (C) 2005 New York Academy of Sciences. doi: 10.1196/annals.1360.011 
and (8) simple temporal codes for pitch capable of accounting for pitches higher than a few hundred hertz have not been found in the auditory cortex. The cortical code for pitch is therefore not likely to be a function of simple rate profiles or synchronous temporal patterns. Studies motivated by interest in the neurophysiology and neuroanatomy of music perception have helped correct longstanding misconceptions about the functional role of auditory cortex in frequency discrimination and pitch perception. Advancing knowledge about the neural coding of pitch is of fundamental importance to the future design of neurobionic therapies for hearing loss.

KEYWORDS: pitch; missing fundamental; psychophysics; periodicity; autocorrelation; auditory cortex; lesion effects; neural coding

\section{INTRODUCTION}

Empirical work on the neurophysiology and neuroanatomy of pitch perception in humans and animals has a long and rich history. The topic is of fundamental interest in neuroscience, for it is well-suited to probing the relationships among (1) physical features of sensory stimuli (e.g., the frequency of a sine tone); (2) perceptual attributes of sensory stimuli (e.g., the pitch of the tone); (3) perceptual constancy across changes in stimulus physics (e.g., pitch constancy across changes in tone intensity); (4) neural coding of stimulus features and perceptual attributes (e.g., action potential firing patterns in single neurons and populations of neurons in the auditory nerve, brain stem, and cortex); and (5) gross and microanatomical mapping of sensory, perceptual, and cognitive functions (e.g., lateralization and localization of regional metabolic changes).

Pitch is the auditory percept associated with the frequency $(f)$ or, equivalently, the period $(T=1 / f)$ of sound wave vibrations in the audible frequency range $(\sim 20-$ $20 \mathrm{kHz}$ ). In Western music notation, a note (e.g., $\mathrm{A}_{4}$ ) symbolizes a distinctive pitch, independent of its loudness, timbre, or other perceptual attributes. The position of the note on the staff and its clef indicate how high or low the pitch is. By convention, the pitch of $\mathrm{A}_{4}$ in the Western scale of equal temperament has the same pitch as a 440-Hz sinusoidal tone (a.k.a. pure tone). The oft-cited, half-century-old definition of pitch by the American National Standards Institute ${ }^{104}$ _ "that attribute of auditory sensation by which sounds may be ordered on a scale extending from low to high"is incomplete, for it implies a single psychological dimension (pitch height). The well-studied perceptual phenomena of octave similarity (pitch chroma) and withinoctave pitch-class hierarchies (e.g., tonic-dominant relationships) establish pitch as a multidimensional percept.

In speech, pitch contrasts convey voiced/unvoiced distinctions, prosodic inflections, and speaker identity. In music, two or more simultaneous pitches comprise harmonic intervals and chords; two or more successive pitches comprise melodic intervals and melodies. Pitch percepts are evoked by a wide range of periodic acoustic signals. Particularly strong pitches are evoked by pure tones and complex tones whose frequencies belong to the same harmonic series (harmonic tones). When frequency components are not harmonically related (inharmonic tones), pitch percepts are weaker, and intervals and chords sound more dissonant.

Stimuli with very different power spectra can produce the same pitch (pitch equivalence or pitch constancy, FIG. 1). The pitch of a harmonic tone corresponds to 

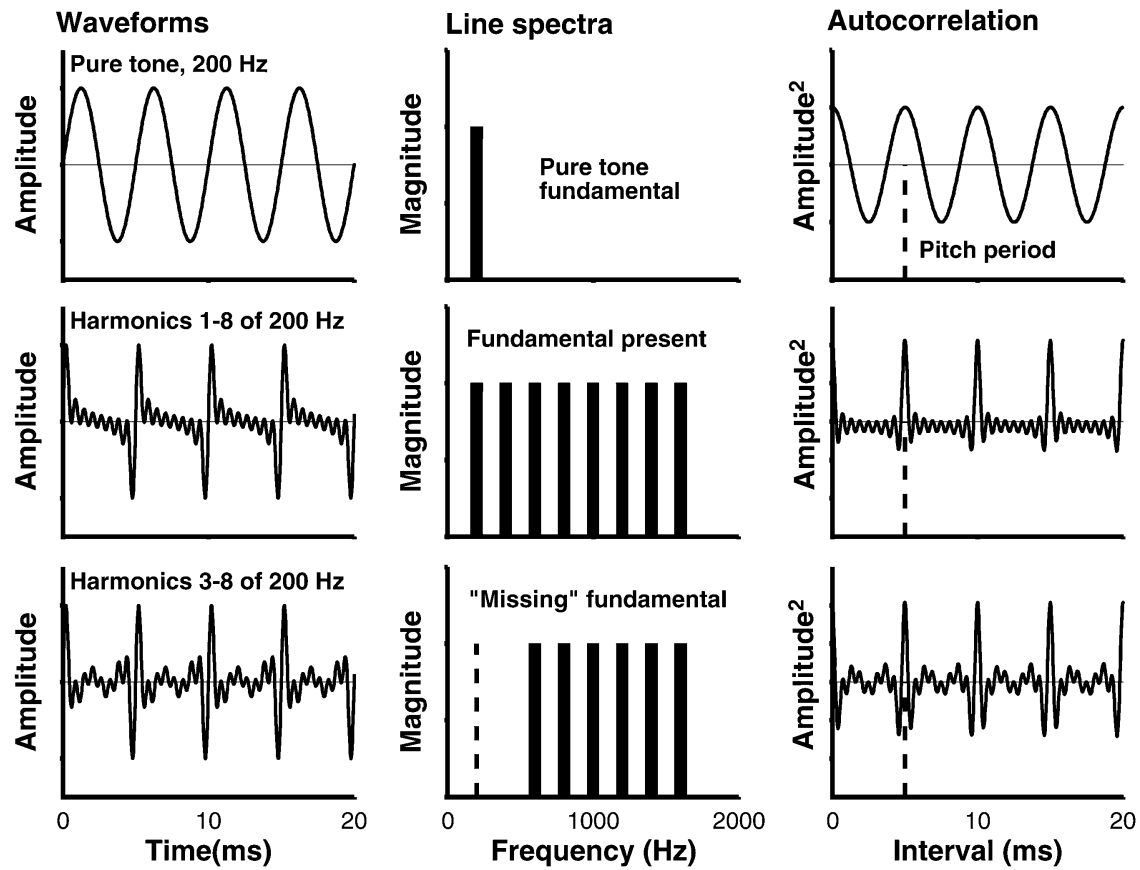

FIGURE 1. Waveforms (left column), line magnitude spectra (middle column), and autocorrelation functions (right column) of a pure tone (top row), harmonic tone with spectral energy at F0 (middle row), and harmonic tone missing F0 (bottom row) that evoke the same pitch. $f=F O=200 \mathrm{~Hz}$. In this example, all frequency components have the same amplitude and phase (sine), but these are not necessary conditions for the three tones' pitch equivalence.

the pitch of a pure tone at the former's F0, even when there is no energy at F0 or lowpass noise masks F0. ${ }^{1-5}$ In the present paper, we use the terms FO pitch when the pitch is evoked by a pure tone or harmonic tone with spectral energy at F0, and missing-FO pitch when the pitch is evoked by a harmonic tone without spectral energy at F0. Both F0 pitch and missing-F0 pitch are perceived by a wide range of animals, including monkeys, ${ }^{8}$ cats, ${ }^{11}$ birds, ${ }^{12}$ frogs, ${ }^{13}$ and fish. ${ }^{14}$ Old World monkeys also perceive octave similarity ${ }^{9}$ and, like rats ${ }^{15}$ and birds, ${ }^{16}$ are sensitive to acoustic features of simultaneous harmonic tones that cue perception of consonance and dissonance in the vertical dimension by humans. ${ }^{7,10}$ On the basis of various theoretical assumptions about the underlying neural processing mechanisms, some authors refer to F0 pitch as spectral pitch, and missing-F0 pitch as virtual pitch, periodicity pitch, residue pitch, and synthetic pitch. However, virtual pitch is virtual only in the sense that F0 is missing in the frequency spectrum of the tone; in the time domain, the fundamental period $\left(\mathrm{T}_{\mathrm{F} 0}=1 / \mathrm{F} 0\right)$ is present as the dominant periodicity in the autocorrelation function of the stimulus (FIG. 1). Missing-F0 pitch is related to Rameau's concept of the basse fondamentale in his Treatise on Harmony. ${ }^{6}$

The capacity to perceive pitch is a basic function of the auditory nervous system that supports melody and harmony perception in music, prosody perception in 
speech, voice recognition, environmental sound recognition, and language acquisition. In light of evidence from comparative neuroscience, ethnomusicology, and developmental neuroscience, it seems reasonable to propose that the underlying auditory mechanisms are innate and that they were necessary (though not sufficient) for the evolution of music among humans.

\section{GROSS NEUROANATOMY AND NEUROPHYSIOLOGY}

Knowledge about the neurophysiology and neuroanatomy of the auditory cortex at the gross, macroscopic level provides insights into the spatial organization of distributed neural systems mediating different aspects of music perception. In addition, it guides the placement of microelectrodes used to study neural coding and neural circuitry at the cellular level.

Most current knowledge about the cortical neuroanatomy of pitch perception is derived from two sources: (1) behavioral experiments with humans and animals who have focal brain lesions; and (2) behavioral experiments and passive stimulation experiments with normal humans that measure changes in blood flow, metabolism, and electrical or magnetic field potentials in normal humans. The strengths of the two methods complement each other.

Lesion effect experiments tell us which gray and white matter structures are necessary for normal performance and allow us to test for functional dissociations. They do not provide information about the full anatomical extent of neurons and axons participating in task performance. In nonhuman primates and other animals, structures of interest can be lesioned selectively by mechanical (e.g., aspiration) or chemical (e.g., ibotenic acid) methods, and the location of the lesion can be pinpointed microanatomically via postmortem inspection of its local histochemical and cytoarchitectonic boundaries as well as its far-reaching effects on the distal axons of damaged neurons (anterograde degeneration) and on the somas of damaged axons (retrograde degeneration). In humans, naturally occurring lesions rarely respect anatomical or physiological boundaries, and in vivo spatial resolution is typically coarse. Because the histopathology of ischemic infarcts and excisions is well circumscribed and homogeneous weeks to years after onset, these types of lesions are better suited for structurefunction studies than intracerebral hemorrhages, Alzheimer disease, brain tumors, and other diseases associated with heterogeneous-focal, multifocal, or diffuse cortical pathology. Knowledge about the anatomical distribution of gross structures that are necessary for the generation of electrical and magnetic field potentials evoked by passive acoustic stimulation has also been gained through lesion studies. Few studies have examined blood flow and metabolism during task performance in patients with ischemic strokes because measurements in the damaged area can be misleading in the face of luxury perfusion, cellular infiltration, and the uncoupling of blood flow and metabolism. In normal human volunteers, analyses of blood flow, metabolism, and field potentials can detect structure-function correlates with good spatial resolution $(\leq 10 \mathrm{~mm}$, depending on the method), provide coarse information about temporal resolution within and across gross structures, and demonstrate the entire distribution of structures that are active during task performance or passive stimulation. However, these methods cannot establish whether any one node in the distributed network of activation plays an essential role in task performance. 


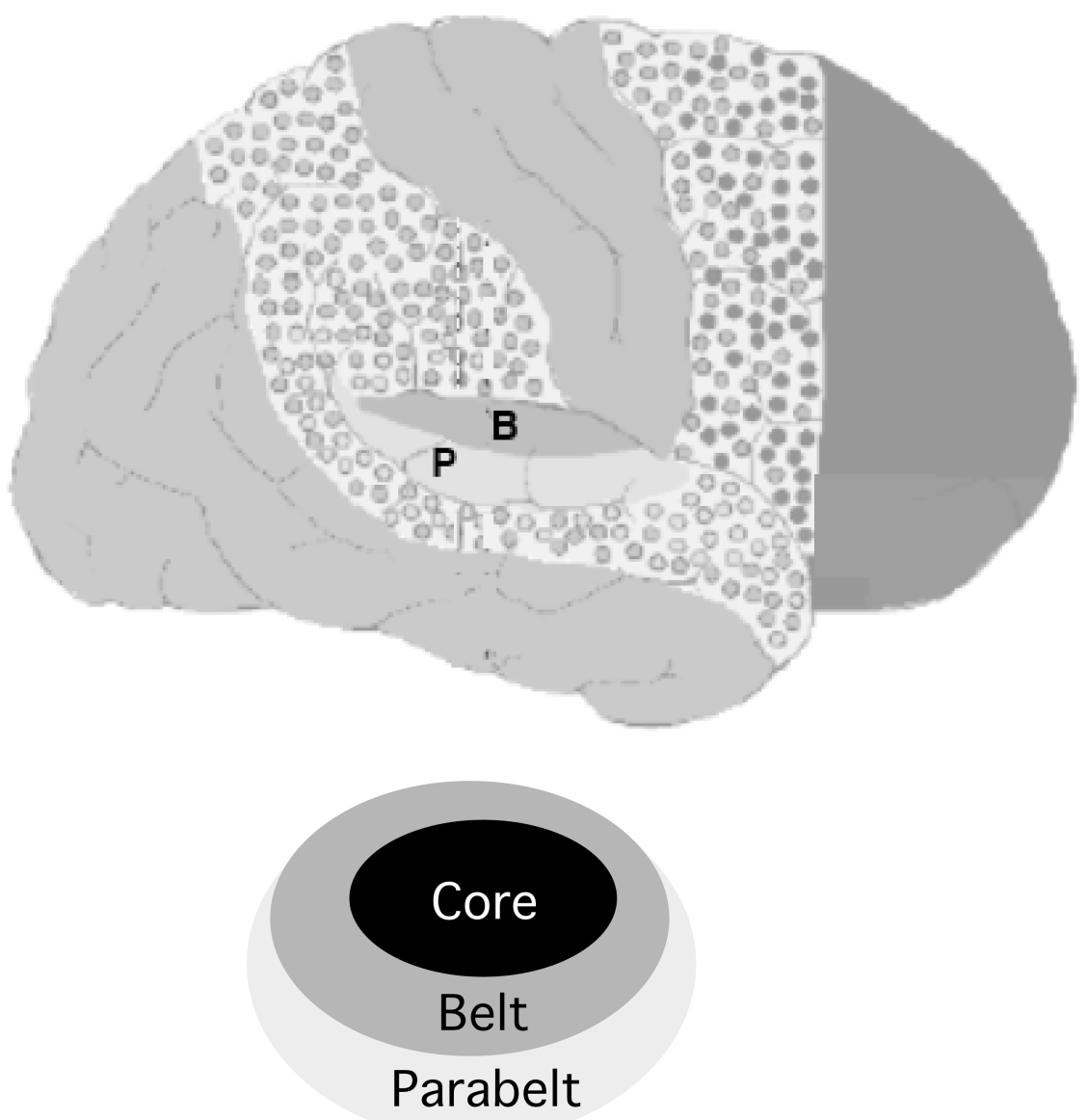

FIGURE 2. (Top) Lateral view, right cerebral hemisphere, human. (Bottom) Schematic of the corresponding core area (black), belt area (dark gray), and parabelt area (light gray) on the superior and lateral surfaces of the right temporal lobe. In humans, apes, and Old World monkeys (e.g., macaques), the core area is buried in the sylvian fissure and cannot be seen on a lateral view. Adapted from Tramo. ${ }^{107}$

The term auditory cortex does not refer to a specific brain structure(s) that can be identified directly via static magnetic resonance images, pathological specimens, or histological sections. By definition, auditory cortex refers to gray matter of the cerebral cortex whose neurons respond to auditory stimuli but not to visual, tactile, or other sensory stimuli. In humans and our close anthropoid relatives, Old World monkeys (including macaques), pathoanatomical, electrophysiological, and metabolic studies collected for over one hundred years have established that the vast majority of auditory cortex neurons are housed in the superior portion of the temporal lobe, inside and below the lateral fissure (FIG. 2). On the superior surface of the superior temporal gyrus (STG, also known as the first temporal convolution, T1), near the 
junction of its anterior two-thirds and posterior third, lies the transverse gyrus(i) of Heschl (TG), prominent in humans, less prominent in apes, and rudimentary in macaques. ${ }^{17-19}$ Most of TG is populated by small, densely packed neurons (koniocortex) and myelinated axons, which bring afferent auditory input from the medial geniculate nucleus (MGN) of the thalamus. TG stains heavily throughout much of its radial and longitudinal extent for cytochrome oxidase, acetylcholinesterase, parvalbumin, Nissl substance, and myelin.

Setting aside differences in physiological and microanatomical criteria and nomenclature that have evolved in the hundred years since Campbell's "auditosensory" and "audito-psychic" dichotomy (c.f. Refs. 17, 20-22), the terms primary auditory cortex (A1) and core area are commonly used to refer to one or more adjacent koniocortical fields containing frequency-selective Layer IV neurons whose topographic organization mirrors the one-dimensional frequency map of the cochlea and whose afferent input comes almost entirely from the ventral division of MGN, which is also populated by frequency-selective, cochleotopically organized neurons. The nomenclature can be confusing: "A1" has been used to refer to the set of all such fields by some authors, including us, and to one specific cochleotopic field within the core area by Hackett and others. A1 is "primary" in the sense that it receives the bulk of short-latency, afferent input from the brain stem's major ascending (lemniscal) pathway via the ventral division of MGN. Around A1 in TG and extending throughout most of the STG is the auditory association cortex (a.k.a., nonprimary auditory cortex). This stretch of gray matter is "associative" in the sense that its neurons synapse with other STG neurons and with neurons in the temporal, frontal, and parietal cortices that respond to stimuli in two or more sensory modalities (multimodal cortex) or that fire without sensory stimulation (supramodal cortex). Many A1 neurons send their axons into a ring of surrounding belt fields of auditory association cortex (FIG. 2), which also receive afferent input from the ascending lemniscaladjunct pathway via the medial MGN, dorsal MGN, and other thalamic nuclei. Like A1 neurons, many belt neurons are frequency selective, that is, they respond over a restricted range of the animal's audible spectrum. Most A1 neurons have spectral bandwidths for on-excitation that are narrower than those of belt neurons. ${ }^{23}$ Many belt neurons send axons into the next surrounding ring of parabelt fields, whose neurons are hard to excite or inhibit with pure tones, a response property shared by the vast majority of cells elsewhere in STG. Neurons in the auditory association cortex are reciprocally connected with multimodal and supramodal neurons in frontal, parietal, and temporal cortices, the basal ganglia, and the cerebellum to form a widely distributed neural system for music cognition.

\section{Effect of Auditory Cortex Lesions on Pure-Tone Pitch Perception}

Despite abundant evidence of neuronal frequency selectivity and cochleotopic organization in A1 and surrounding fields in multiple animal species, the regnant view among twentieth-century neuropsychologists, neurophysiologists, and neuroanatomists held that the auditory cortex was not necessary for normal performance on pure-tone pitch discrimination tasks. In 1963, on the basis of unpublished selective-ablation experiments in cats, Guttman and Diamond ${ }^{24}$ argued, "tonotopic organization at the cortical level is not necessary for the perception of tones." In 1975, in their authoritative review of animal and human lesion effects in the Handbook of 

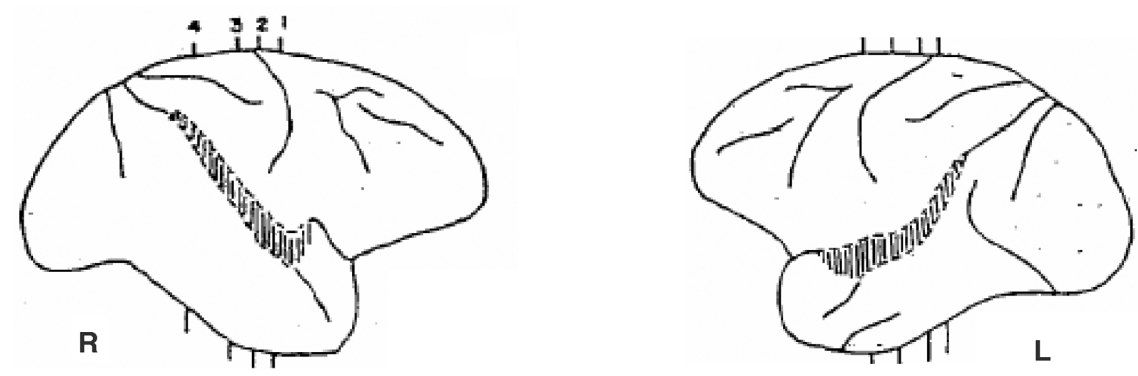

FIGURE 3. Lateral view, right (R) and left (L) cerebral hemispheres of a rhesus monkey (Macaca mulatta), whose gross-microanatomical correlates are similar to those of other Old World monkeys but not New World monkeys. The dark area in each hemisphere marks the ablation site in the lateral surface of STG; this lateral view does not show the lesions made in the superior surface of STG, which houses A1 in Old World monkeys, apes, and humans. Curved lines within each hemisphere are the major fissures and sulci of the cerebral hemispheres. Vertical lines above and below each hemisphere indicate the locations of postmortem coronal sections that were inspected macro- and microscopically for hemispheric and thalamic lesions. Adapted from Evarts. ${ }^{27}$

Sensory Physiology, ${ }^{24}$ Neff, Diamond, and Casseday concluded, "frequency discrimination may be learned or relearned after bilateral lesions involving all or nearly all of primary auditory cortex in animals such as the cat and monkey and in human patients." The Neff doctrine had a profound influence on current opinion about structure-function and physiological-perceptual correlates throughout the cognitive neuroscience boom of the late twentieth century. For example, after finding a deficit in missing-F0 pitch discrimination in a subpopulation of right temporal lobectomy patients, Zatorre ${ }^{38}$ stated, "simple frequency discrimination (i.e., with pure tones, or with complex tones when the fundamental [frequency] is present) is not permanently disrupted even by large bilateral lesions of auditory cortex." Scrutiny of the experiments in Old World monkeys and humans on which these claims were based yields important lessons about methodology that have heuristic value for future work in the field. (For a critical review of selective-ablation studies in cats, see Refs. 25 and 26).

Among primate lesion effect studies cited by Neff et $a .^{24}$ was Evarts, ${ }^{27}$ condition-ablation experiment in macaques ${ }^{27}$ (FIG. 3). A go/no-go, one-interval, two-alternative, forced-choice, operant-conditioning task required each monkey to respond differentially to a $350 \mathrm{~Hz}$ pure tone and a $3500 \mathrm{~Hz}$ pure tone of the same duration and similar intensity. After near-complete bilateral ablations of superior temporal cortex, one monkey (M-3) needed 600 trials to learn the task and reach the response criterion of $80 \%$ accuracy over 50 consecutive trials. Two other monkeys (M-19 and M-20) were studied preoperatively as well as postoperatively. Before surgery, one monkey needed 450 trials and the other 700 trials to reach the response criterion. After bilateral ablation of TG and all but a small anterior portion of STG, one monkey reached the response criterion in the first 50 trials, and the other was able to relearn the task with less training than it needed preoperatively. Evarts remarked that the results were "difficult to reconcile with the strict tonotopic organization" of primate A1 and speculated that small remnants of remaining auditory cortex were "of great 
functional importance." However, interpretation of the results is confounded by a methodological flaw: the task failed to assess pure-tone pitch discrimination anywhere near psychophysical threshold. The frequency difference $(\Delta \mathrm{f})$ between the tones was $3150 \mathrm{~Hz}$, and the Weber fraction was $164 \%$ [ $\Delta \mathrm{f} /$ mean $\Delta \mathrm{f}) \times 100]$. In other words, the behavioral task was too insensitive, so the experiment was biased in favor of supporting the null hypothesis.

In fact, clinical case reports, audiological assessments, and neuropsychological experiments on F0 pitch throughout the nineteenth and twentieth centuries suffered from the same methodological flaw. For example, the oft-cited cases of Jerger and colleagues $^{28,29}$ and Zatorre ${ }^{38}$ were examined using pure-tone $\Delta \mathrm{f}^{\prime}$ 's and harmonictone $\Delta \mathrm{F} 0$ 's corresponding to Weber fractions of $\sim 40 \%$, about 40 times the normal threshold (Weber fraction $\sim 1 \%$ ). ${ }^{30}$ In addition, the sensitivity, specificity, and spatial resolution of their anatomical methods precluded precise definition of lesion sites and sizes. Neurologists and other physicians never carry tuning forks that are less than an octave apart (Weber fraction $=67 \%$ ), and when keyboards are used to test patients in the hospital or laboratory, the minimum $\Delta \mathrm{F} 0$ they can test is constrained by the Western scale of equal temperament, which has a minimum step size of approximately $6 \%$. In general, the test method used by Evarts, Jerger, and many others - the method of constant stimuli-is not well suited to measuring psychophysical thresholds, because the stimuli are "canned:" they are designed and generated before performance is tested. This makes it difficult to place observations near each listener's threshold unless pilot studies can provide useful constraints.

Experiments carried out in recent years at separate laboratories with different neurological patients, many of which were motivated by interest in the neuroanatomy of music perception, have rendered the Neff doctrine untenable. Together, the results have forced a fundamental change in current opinion about auditory cortex function and a reappraisal of the functional relevance of neuronal frequency-selectivity in A1.

In 1989, my colleagues and I began a series of experiments on pitch, harmony, and melody perception with a middle-aged, ambidextrous man, MHS, who has chronic bilateral auditory cortex infarcts. His subjective complaints of impaired music, speech, and environmental sound perception were precipitated by his second stroke, which was very small but unfortunately placed in his left TG and posterior STG (FIG. 4). His previous right-sided infarct involved most of the right middle cerebral artery territory, including TG, STG, and multiple temporal, frontal, and parietal gray and white matter structures. The first clue that he had impaired pitch discrimination came from the Seashore Measures of Musical Talents Pitch Discrimination Test, ${ }^{31}$ which measures the accuracy of pitch direction discrimination using a two-interval, two-alternative, forced-choice paradigm and the method of constant stimuli. The test contains five blocks of ten trials in which $\Delta \mathrm{f}$ decreases over successive blocks. With stimuli presented over loudspeakers at a comfortable listening level, MHS's response accuracy fell to chance as $\Delta$ f decreased. ${ }^{32}$ We subsequently used an adaptive procedure and well-calibrated pure tones to measure $\Delta \mathrm{f}$ thresholds for pure-tone pitch perception. ${ }^{30,33}$ In one experiment, MHS was asked to judge whether the pitch of the second tone was higher or lower than the pitch of the first tone (pitch direction discrimination); in another, he was asked to judge whether the pitch of the second tone was the same as or different from the pitch of the first tone (pitch change detection). Normal and patient controls performed well on both tasks [Weber 


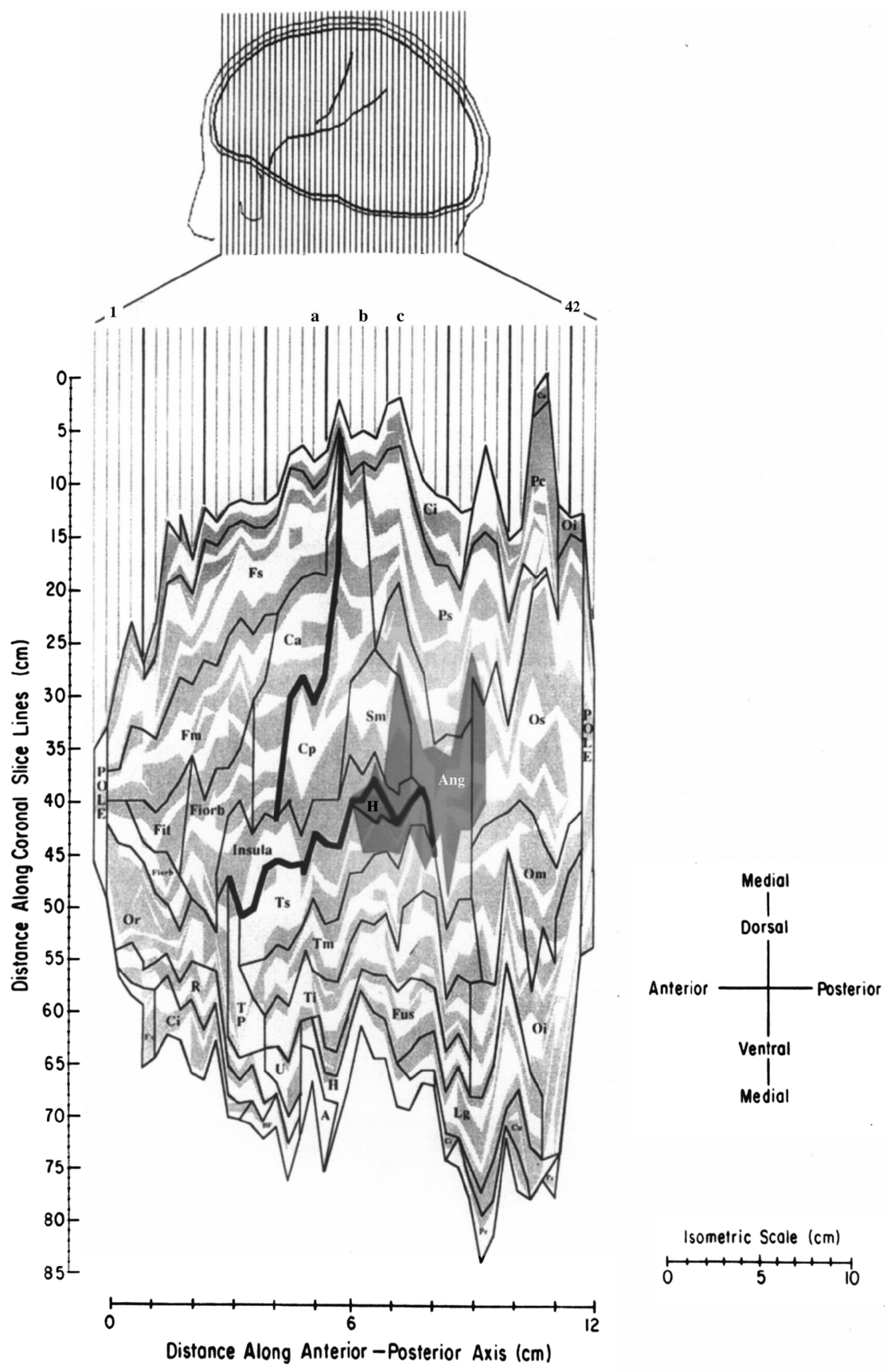

FIGURE 4. Flat map of the unfolded surface of the left cerebral cortex of case MHS (a.k.a., case $\mathrm{A} 1+)$ reconstructed from MRIs. Bold lines indicate the lateral and central fissures. The dark gray area near the lateral fissure marks the infarct. Light gray areas throughout the map indicate intrasulcal surfaces, white areas pial surfaces. Adapted from Tramo et al. ${ }^{105}$ 


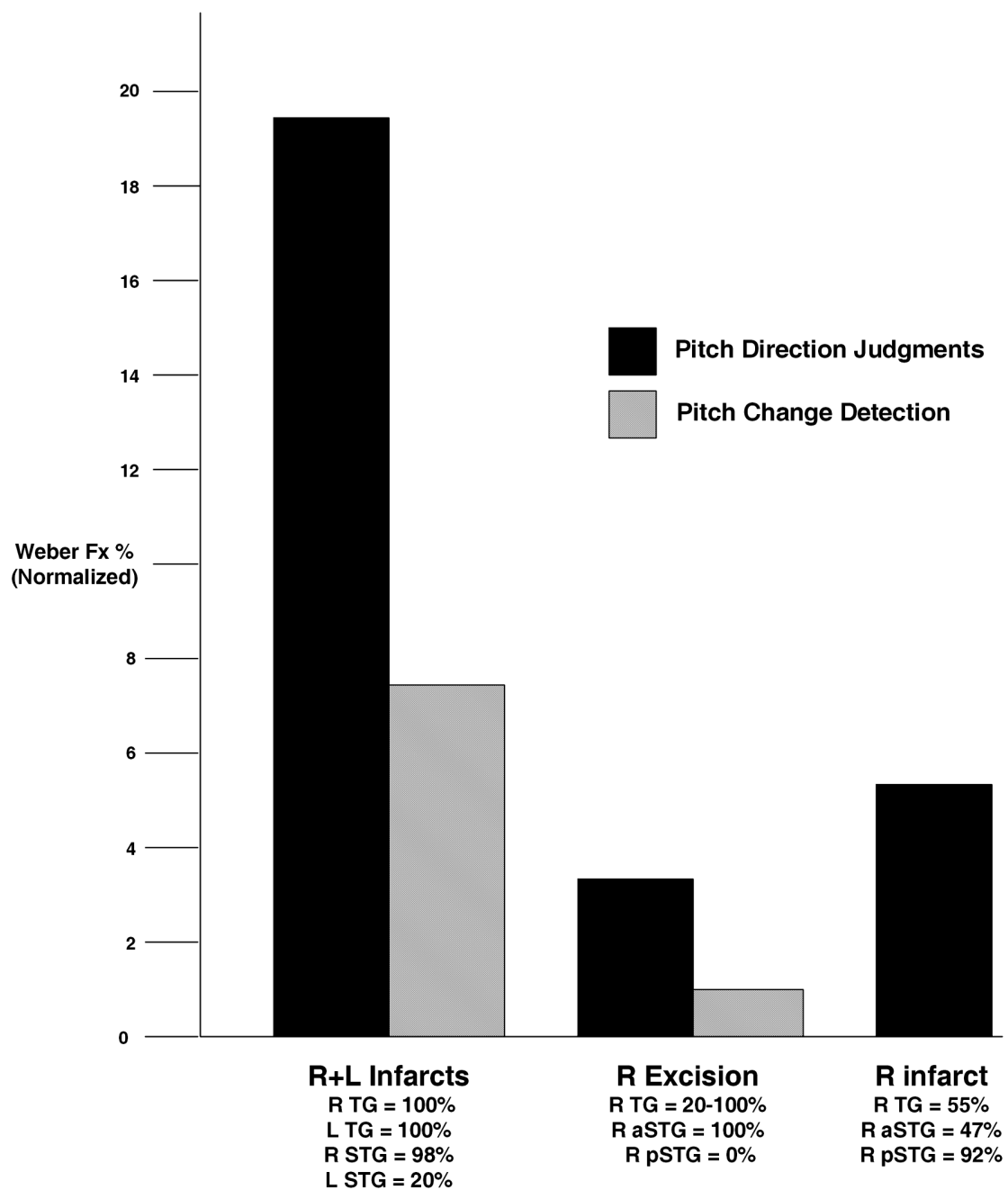

FIGURE 5. Bar graph showing Weber fractions for pitch direction discrimination (higher-lower judgments, black bars) and pitch change detection (same-different judgments, gray bars) in patients with auditory cortex lesions. (Left bars) Case MHS. (Middle bars) Mean thresholds from six temporal lobectomy patients with partial right TG and partial STG lesions reported by Johnsrude et al..$^{35}$ (Right bars) Case WKF. Because the tasks used to measure thresholds in MHS, WKF, and the temporal lobectomy patients differed (albeit slightly), normalized Weber fractions were computed by dividing patient Weber fractions by the mean Weber fraction measured in separate groups of normal controls. 
fraction $\sim 1 \%$ at $1 \mathrm{kHz}$, intensity $=40 \mathrm{~dB}$, sensation level (SL) at each ear, tone duration $=500 \mathrm{~ms}$, interstimulus interval (ISI) $=200 \mathrm{~ms}$. MHS's performance differed from that of controls in two ways: (1) his Weber fractions were seven or more times higher than those of controls on both tasks; and (2) his Weber fraction for pitch direction discrimination was twice that for pitch change detection (FIG. 5). Kazui et al. ${ }^{34}$ also reported elevated thresholds for pitch change detection in a stroke patient with bilateral TG and left STG lesions; pitch direction discrimination was not tested.

The results of recent condition-ablation experiments in Old World monkeys are consistent with the effects of bilateral auditory cortex lesion effect in humans. Harrington et al. ${ }^{41}$ reported that $\Delta \mathrm{f}$ thresholds for pitch change detection in macaques with bilateral complete TG lesions and near-complete STG lesions were more than twice the $\Delta \mathrm{f}$ thresholds in normal macaques.

Comparisons between the effects of bilateral versus unilateral auditory cortex lesions on pitch perception may shed light on hemispheric specialization and interhemispheric integration. Divenyi and Robertson ${ }^{36}$ reported elevated pitch change detection thresholds in four patients with right hemisphere strokes. Pitch direction discrimination was not tested, and lesion localization was not reported. Johnsude et $a l .{ }^{35}$ used an adaptive procedure and a two-interval two-alternative forced choice paradigm to measure $\Delta f$ thresholds for pure-tone pitch change detection and pitch direction discrimination in epilepsy patients with anterior temporal lobectomies $(f=$ $800-1200 \mathrm{~Hz}$; intensity $=75 \pm 2 \mathrm{dBA}$ A.U.; ISI = $1 \mathrm{~s}$ ). On the direction discrimination task, seven of eight patients with lesions involving right TG (as well as right anterior STG and other temporal lobe structures) had Weber fractions that were three or more times greater than normal (FIG. 5); the mean performance of lobectomy patients with left- or right-sided lesions that spared right TG was within normal limits. By contrast, on the change detection task, most right TG patients, like other patients, had normal Weber fraction. We have also observed elevated Weber fractions for pitch direction discrimination following a partial right TG lesion in stroke patient WKF, a right-handed musician whose chronic infarct also involves right posterior STG, and neighboring gyri but spares much of right anterior STG (FIGS. 5 and 6). Threshold elevations were greater when pure tones were presented to the ear (left) contralateral to the auditory cortex lesion. Thresholds for pitch change detection have not yet been tested.

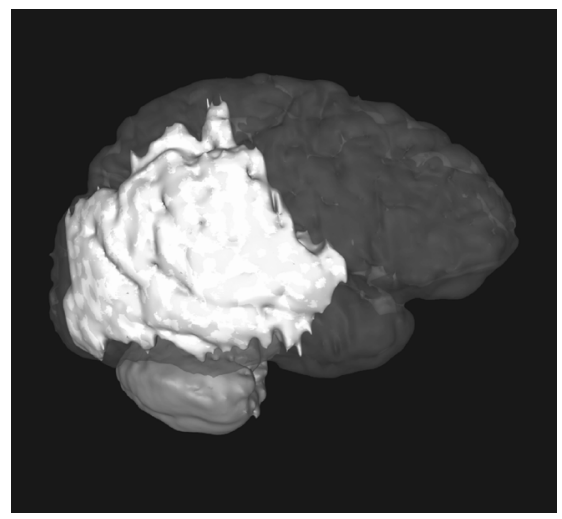

FIGURE 6. 3-D MRI reconstruction of the right cerebral hemisphere of case WKF. The light area marks the infarct. 
Taken together, the effects of bilateral and unilateral auditory cortex lesions on pure-tone pitch perception in humans and macaques raise the following hypotheses: (1) frequency-selective neurons in both right and left A1 contribute to pitch change detection and pitch direction discrimination; (2) the contributions of right and left A1 neurons to pitch direction discrimination are additive; and (3) frequencyselective neurons in right A1 are necessary for normal pitch direction discrimination in most right-handed adults independent of musicality.

\section{Effect of Auditory Cortex Lesions on Harmonic-Tone Pitch Perception}

Bilateral lesions of auditory cortex elevate $\Delta \mathrm{F} 0$ thresholds for pitch direction discrimination whether $\mathrm{F} 0$ is present or missing in a harmonic complex tone. ${ }^{37}$ The magnitude of the $\Delta \mathrm{F} 0$ threshold elevation appears to be proportional to the $\Delta \mathrm{f}$ threshold elevation obtained with pure-tone stimuli. This suggests that missing-F0 impairments may be attributable to derangements in "low-level" processes (e.g., frequency resolution) rather than higher-level processes (e.g., harmonic template matching).

Studies of unilateral lesion effects on F0 pitch perception were published by three laboratories between 1988 and 1990. Their results conflict with respect to the contribution of right auditory cortex when energy is present at F0. Sidtis and Volpe's harmonic-tone experiments in stroke patients used a dichotic-diotic match-to-sample pitch recognition task and the method of constant stimuli. ${ }^{42}$ Deficits in F0 pitch perception were found in a population with right but not left hemisphere strokes. The site and size of the lesions were not reported. Zatorre's ${ }^{38}$ harmonic-tone experiments in epilepsy patients with temporal lobectomies used a binaural, two-interval, twoalternative forced-choice task and the method of constant stimuli. ${ }^{38} \mathrm{~A}$ dissociation between missing-F0 pitch (impaired) and F0 pitch (spared) was found in a population of patients with right-sided excisions involving anterior STG and TG. However, task difficulty differed for the two stimulus conditions. Harmonic tones in the F0 pitch task contained nine harmonics; those in the missing-F0 pitch task contained three or four. The resulting difference in stimulus pitch strength rendered the missing-F0 pitch task harder than the F0 pitch task and thus confounds a straightforward interpretation of the observed dissociation. Soon thereafter, Robin et al. ${ }^{39}$ reported elevated $\Delta \mathrm{F} 0$ difference threshold for $\mathrm{F} 0$ pitch change detection in five stroke patients with right, but not left, STG and/or TG lesions. ${ }^{39}$ Their experiment employed square-wave stimuli presented binaurally via loudspeaker (i.e., harmonic tones containing odd harmonics, including F0, that decrease in intensity with increasing $f$; duration $=200 \mathrm{~ms}$, ISI $=500 \mathrm{~ms}$, intensity $=70 \mathrm{~dB}$ SPL), an adaptive procedure, and a three-interval, two-alternative, forced-choice task that required listeners to judge which of the two comparison tones in the second and third stimulus intervals differed from the standard tone in the first interval. In summary: (1) the results of Robin et al. and those of Sidtis and Volpe agree that right-sided lesions impair F0 pitch perception in right-handed adults; (2) Zatorre's results indicate that right anterior STG and partial TG lesions impair missing-F0 pitch perception in right-handed adults; and (3) methodological differences hamper comparisons among the three studies.

The results of the most recent temporal lobectomy population study, ${ }^{106}$ which employed a two-interval "same-different" forced-choice task and the method of constant stimuli, have been interpreted as evidence that right STG and TG excisions 
do not impair performance on same-different F0 pitch judgments of harmonic tones with 12 harmonics, even when there is little spectral energy at F0 relative to that at higher harmonics (binaural intensity $=75 \mathrm{~dB}$ SPL, tone duration $=500 \mathrm{~ms}$, ISI $=$ $100 \mathrm{~ms}$ ). However, inspection of the population data (Ref. 106, FIG. 5, p. 1621, same timbre conditions) raises the possibility that patients with right excisions performed worse than all or almost all of those with left excisions and worse than most normal controls.

Using a two-interval, two-alternative, forced-choice task and an adaptive procedure, we measured F0 difference thresholds for pitch direction discrimination following unilateral infarction of right TG, STG, and adjacent gyri in Case WKF (FIG. 6). Our preliminary results obtained with missing-F0 tones raise the possibility that right auditory cortex mechanisms mediating the temporal processing of envelope periodicities generated by combinations of unresolvable high harmonics are dissociable from those mediating the spectral and/or temporal processing of individual, resolvable harmonics.

Acknowledging that the sum total of patients and nonhuman primates with focal lesions involving different sides and subdivisions of auditory cortex is small, and that replication of structure-function correlates by different laboratories remains in short supply, we tentatively interpret the results of lesion experiments employing tones or vocalizations with F0s, intensities, and durations typical of notes anchoring melodies in traditional Western vocal and instrumental music ${ }^{30,33,35-38,40-43,106}$ as follows: (1) frequency-selective neurons in A1 and adjacent areas in right and left TG and STG play critical, additive roles in our ability to detect a small change in pitch between two successive pure tones or harmonic tones; (2) right-sided neurons play a critical role in our ability to perceive the direction of a pitch change between two successive pure tones or harmonic tones; (3) left-sided neurons play an additive role in our ability to perceive the direction of a pitch change between two successive pure tones or harmonic tones; (4) right auditory cortex mechanisms mediating perception of F0 pitch versus missing-F0 pitch are neurologically dissociable; and (5) right auditory cortex mechanisms mediating spectral versus temporal processing of harmonic tones are neurologically dissociable.

\section{Gross Physiological Changes during FO Pitch Perception}

Measurements of cortical blood flow and metabolism during pitch perception also provide insights into structure-function relationships at the gross anatomical level. ${ }^{48}$ Zatorre et al. $^{46}$ measured changes in cortical blood flow while ten young right-handed adults listened to pairs of consonant-vowel-consonant syllables differing in pitch and final consonant. ${ }^{46}$ The size of the F0 difference between syllables was not given. When the volunteers completed 40 trials of a go/no-go two-interval, two-alternative forced-choice task that required them to respond differentially to the direction of the pitch change between syllables, blood flow was significantly higher in regions of right anterolateral frontal cortex, left posterodorsal frontal cortex, and medial occipital cortex than when the volunteers listened passively to the syllable pairs and performed a simple, repetitive task. Compared to lying still with minimal acoustic stimulation (ambient room noise), passive stimulation with syllables or amplitude-modulated noise during repetitive finger movements increased blood flow in portions of the left TG, right STG, left STG, and many other gyri. While large-scale 
differences in stimulus design, task requirements, and brain measures preclude a straightforward comparison of these structure-function correlates with those observed in lesion effect studies, the results (1) demonstrate that widespread regions of the cerebral cortex are active during acoustic stimulation and task performance; (2) neither support nor refute Zatorre's claim ${ }^{38}$ that lesions of the right temporal lobe have no effect on complex-tone pitch perception when energy is present at F0, contrary to the authors' assertion that the observed right frontal activation during F0 pitch judgments was predicted on the basis of those results; and (3) suggest that supramodal systems involved in decision making and working memory during F0 pitch processing are lateralized to the right anterolateral frontal cortex.

Two recent fMRI studies suggest that the magnitude and spatial distribution of neural activity in A1 and adjacent auditory association cortex vary with pitch independent of stimulus physics. ${ }^{49,50}$ For example, passive stimulation with harmonic tones that have a strong pitch evokes greater activation in left and right anterolateral TG and adjacent left STG than harmonic tones that have a weak pitch. Sequences with pitches that vary over time evoked greater activation in subregions of right STG than monotonic pitch sequences. ${ }^{49}$ Magnetoencephalographic localization of responses evoked by tones with, versus without, spectral energy at F0 raise the possibility of a place representation of pitch in $\mathrm{A} 1.44,45$

\section{NEURAL CODING OF PITCH}

What are the neural representations and computations that give rise to pitch and harmony? An adequate theory needs to explain how the brain achieves high precision for pitch discrimination (pure-tone and complex-tone difference thresholds $[\sim 1 \%$ in naive listeners $]$ and inharmonicity detection $[\sim 1 \%$ shift of one harmonic $\left.{ }^{51}\right]$ ). It must also account for pitch equivalence despite differences in spectrum and level invariance over large dynamic ranges (within 1-2\% across $>60 \mathrm{~dB}$ SPL). ${ }^{4,52}$ In view of pitch equivalence and level invariance, the breakdown of tonotopy and nonmonotonic changes in discharge rates as a function of spectrum and intensity ${ }^{53}$ confound a straightforward rate-place theory for pitch at the level of the auditory cortex.

\section{Neurocomputational Mechanisms for Pitch Processing}

Theories of pitch can be divided into two types: spectral-pattern models and temporal models. Spectral models first infer the pure-tone frequency components present in a sound from neural activity patterns, usually population rate-place profiles, and then carry out a harmonic analysis on that pattern. Connectionist networks can form harmonic templates if they have inputs that are highly frequency selective and well behaved in other ways. ${ }^{54,55}$ Some evidence for the kinds of sharp frequency tuning and harmonic-combination selectivity that these models require has been observed in cortical neurons in barbiturate-anesthetized cats ${ }^{56}$ and unanesthetized bats. ${ }^{57-59}$ However, harmonic-combination selectivity has only been seen at best frequencies (BFs) well above those relevant for virtual pitches (BFs $>5 \mathrm{kHz}$ ). Another difficulty for spectral pattern models is that they cannot explain strong virtual pitches that can be evoked by sets of harmonics $(n>6)$ that are not resolved perceptually. 4,60 
Temporal models posit that neurons represent information about stimulus periodicities in the fine-timing of their discharges, specifically in the time intervals between spikes ("interspike intervals"). Currently, the strongest neurocomputational models for pitch and harmony are based on population-wide distributions of allorder interspike intervals in the auditory nerve. ${ }^{61-63}$ Features of these representations correspond very well to many aspects of pitch perception: missing-F0 pitch; pitch equivalence; level and phase invariance; pitch strength (salience); unresolved harmonics, pitch shift of inharmonic tones (de Boer's rule); pitch ambiguity; the dominance region for pitch; Rameau's basse fondamentale; and the consonance and dissonance of musical intervals. Patterns of interval peaks in population-interval distributions are similarly capable of representing vowel quality. ${ }^{63-65}$ Since phase locking and interspike interval information decline above $4 \mathrm{kHz}$, these models also

A

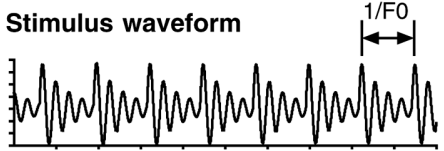

B

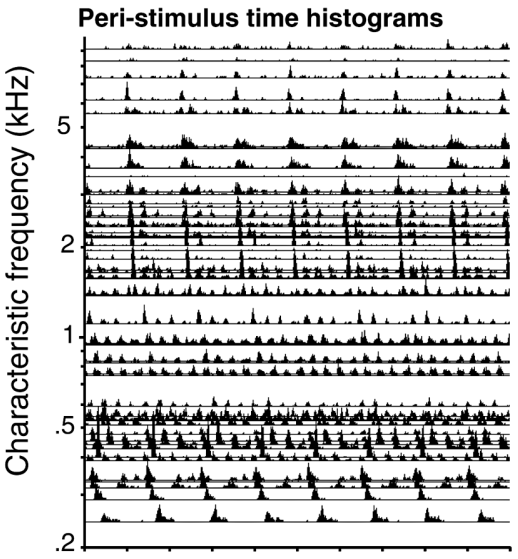

D Stimulus autocorrelation function 1/FO pitch period

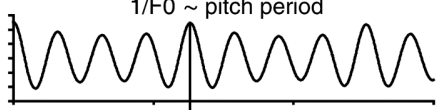

E Interspike interval histograms

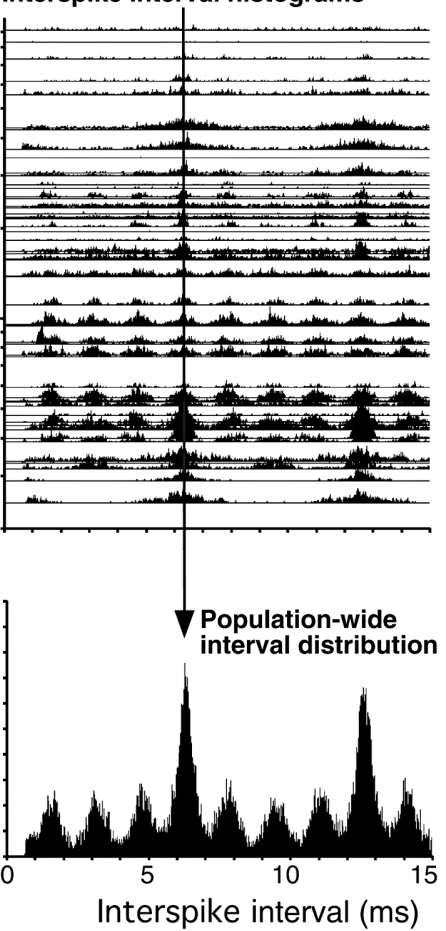

FIGURE 7. Temporal coding of pitch in the cat auditory nerve. (Left) Peristimulus time histograms of auditory nerve fibers in response to a vowel-like stimulus presented at moderate sound pressure level. (Right) Interspike interval distributions produced in single fibers and across the fiber population, whose features correspond closely to the pitches that are heard. ${ }^{62}$ 
explain the decline of pure-tone frequency discrimination at higher frequencies ${ }^{66}$ and the existence region for musical tonality (up to about $4 \mathrm{kHz}$ for octave matching and melodic recognition) (FIG. 7).

\section{Coding Transformations in the Auditory Pathway}

The spike timing information about pitch and harmony must either be analyzed in lower auditory stations in brain stem and midbrain, where it is abundantly available, or be transformed into some other form for processing in higher, thalamocortical centers.

The functional role of the auditory cortex is very different in these two models. In the first, fine-grained, temporal representations of pitch would exist only in lower stations. Here the cortex, through descending control paths, would dynamically organize lower centers to set up temporary, task-dependent circuits for pitch detection, discrimination, and recognition tasks. Cortical lesions would have the effect of degrading the control apparatus necessary for precise pitch distinctions. Relatively little is currently known about short-term bottom-up/top-down circuit dynamics in the auditory pathway, largely because most single-unit studies to date have been carried out in anesthetized animals. In the coding transformation conception, fine-grained pitch-related information is retained at higher levels, albeit in different form. A coding transformation would entail an orderly conversion of all-order interspike interval information into representations based on firing rate, spike latencies, more complex temporal patterns, interneural spike synchronies, and the like. In the transformational view, cortical lesions would have the effect of degrading neuronal populations that are involved in both conveying and processing pitch-related information.

Rate-based "periodicity detectors" situated in the midbrain have often been proposed as a plausible time-to-rate transformation. ${ }^{67-71}$ While many neurons in the auditory pathway respond best to particular periodicity ranges (i.e., they have bandpass modulation-transfer functions), coding schemes based on periodicity-to-rate transformations are at odds with psychophysical observations: (1) modulation tuning is too coarse to support fine-grained pitch discrimination; (2) it is not level invariant: ${ }^{72,73}$ (3) it cannot by itself account for pitch equivalence, since pure tones are unmodulated; and (4) it cannot account for the pitch of inharmonic tones, since modulation detectors, unlike pitch percepts, follow envelopes rather than fine structure when the two conflict. ${ }^{1,74}$ Psychophysical data on the pitch of inharmonic tones strongly suggest a central temporal autocorrelation analysis rather than a modulationor envelope-based one. Licklider's 1951 time-delay (TDNN) autocorrelation network could carry out the right time-place transformation, but no units with the requisite fine comb-filter tunings have been found at brain stem levels. Modulation tunings are seen in many units in the auditory thalamus and cortex,${ }^{75}$ but they are generally very coarse compared to pitch discrimination thresholds. While a few cortical units have best modulation frequencies (BMFs) in the periodicity pitch range $(50-1000 \mathrm{~Hz})$, the vast majority have BMFs well below this range (typically $4-16 \mathrm{~Hz}$ ).

\section{Central Temporal Codes}

Within the central auditory system, evidence that pitch and harmony are encoded in the all-order interspike interval distribution is abundant in all three major divisions of the cochlear nucleus. ${ }^{63,76-78}$ The existence of binaural periodicity pitches ${ }^{79}$ 
suggests that mechanisms for pitch analysis must exist above the level of the superior olive, where timing information from both ears is preserved and combined. Intervalbased representations of pitch and harmony at the level of the inferior colliculus are also possible. ${ }^{80,81}$ Considerable phase-locking can be seen in the medial geniculate nucleus of lightly anesthetized animals: $10-20 \%$ of single units have synchronization indices of $\geq 0.3$ to $1-2 \mathrm{kHz}$ tones. ${ }^{82}$ Neurons with these properties would almost certainly support fine pitch discriminations typical of human listeners. ${ }^{66}$

However, the extent to which these kinds of temporal representations can support pitch and harmony in the auditory cortex is much less clear. Averaged cortical potentials show periodicities up to several hundred hertz in response to click trains. ${ }^{83-87}$ This, of course, is a pale remnant of the temporal information available in the auditory nerve and brain stem, where periodicities up to 4 or $5 \mathrm{kHz}$ are seen. As one ascends the auditory pathway, both phase-locking and average discharge rates decline, and, as a consequence, it becomes increasingly difficult to find any units that produce a stimulus-related interspike interval pattern. If a cortical temporal code for pitch exists, it must be present in an asynchronous, covert, and/or sparse form, or it would have been observed by now with the methods at hand.

A sparse interval code is difficult to rule out completely. Since the numbers of neurons at higher stations increase dramatically, the same quantity of fine-timing information seen at lower stations might be more sparsely distributed across increasingly larger populations (sparse temporal code). De Ribaupierre and coworkers ${ }^{88}$ found 2 of 179 neurons in A1 of unanesthetized cats that were capable of phase locking to click train F0s throughout the entire existence region for missing-F0 pitch $(50-800 \mathrm{~Hz})$. While $<2 \%$ is a small number in his sample, macaque A1 and surrounding areas contain over 10 million neurons; ${ }^{89}$ consequently over 100,000 neurons may be available for interspike interval coding of missing-F0 pitch.

Could a central time code exist in covert form? For example, pitch-related timing information could be multiplexed with spike patterns that encode other aspects of the stimulus, such as timbre, roughness, location, loudness, and perceptual grouping, in which coding of each perceptual dimension relied on a different aspect of the population response (average rates, compactness of spike latency distributions, interval statistics, spike pattern correlations). Most of the common methods for cortical spike train analysis would miss temporal patterns of spikes embedded amid other spikes (multiplexed temporal codes), patterns that appeared at different times in the neuronal response (gated temporal code), patterns that involved interspike intervals be-

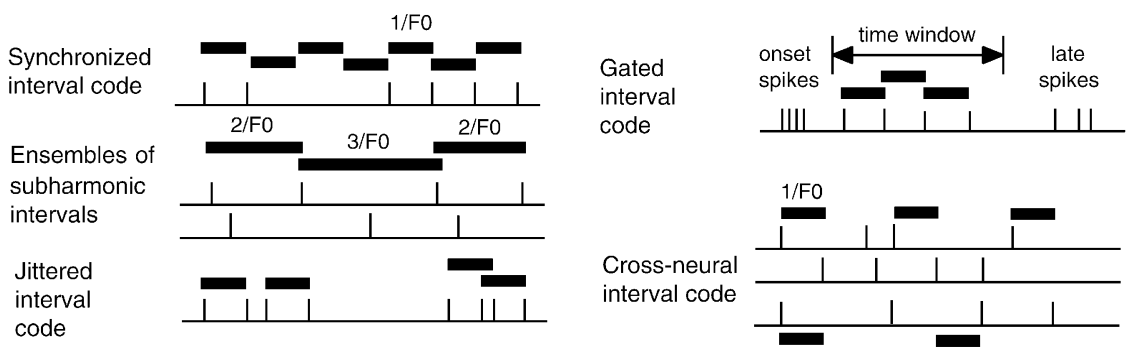

FIGURE 8. Possible synchronous and asynchronous coding schemes for encoding pitch-related periodicity information in spike correlation patterns. 

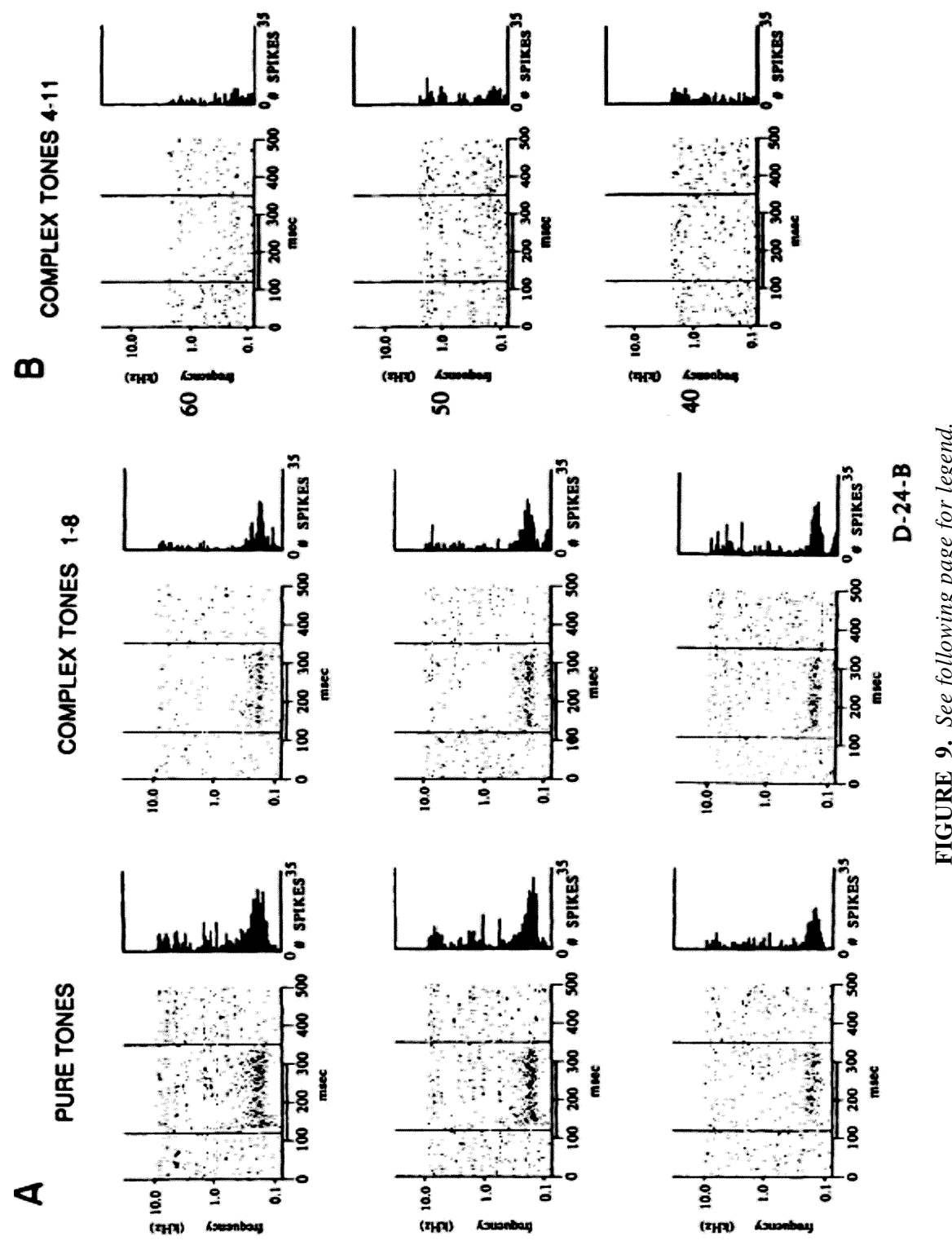
tween spike trains of multiple neurons (ensemble spike correlation code), patterns that were not rigidly time locked to the stimulus onset (asynchronous, jittered patterns), and periodicities related to subharmonics of F0 (FIG. 8). Although evidence for these and other alternative coding schemes has been found elsewhere, ${ }^{90-100}$ most of these possibilities have yet to be explored in the auditory cortex.

\section{Rate-Based Cortical Representations of Pitch}

In recent decades most auditory neurophysiology has focused on characterizing input-output behavior of individual neurons rather than searching for response correlates of perceptual dimensions, distinctions, and invariances. In the latter strategy, one keeps the percept constant and analyzes for underlying commonalities in response patterns. Only one single-unit study in unanesthetized Old World monkeys has systematically investigated whether frequency-selective neurons change their firing rate as a function of pitch, independent of stimulus spectrum. ${ }^{101}$ Stimuli included pure tones and harmonic tones with and without energy at F0, although Schwarz and Tomlinson found frequency-selective neurons in and around A1 that increased their discharge rates during stimulation with pure tones and harmonic tones with F0s near BF but not with harmonic tones missing F0 near BF (FIG. 9). Furthermore, no neurons displayed discharge rate profiles capable of resolving harmonic separations less than $300 \mathrm{~Hz}$. In a much more limited study in anesthetized macaques, Riquimaroux and Hashikawa ${ }^{102}$ reported that 15 of 15 neurons in A1 showed increases in firing rate to pure tones and harmonic tones with and without $\mathrm{F} 0$ at BF. It is not clear whether combination tones at BF or total stimulus spectral energy in the cell's frequency receptive field contributed to these results.

Evidence of a rate-based neural code for pitch in the auditory cortex of a New World monkey was recently reported by Bendor and Wang. ${ }^{103}$ Peaks in the discharge rate profiles of 53 neurons tuned to low pure-tone frequencies in and around A1 were observed for harmonic-tone F0s and missing F0s near BF. Pitch equivalence might therefore be explained through the common activation of such units by sets of spectrally diverse sounds that have the same periodicity. Whether these neurons were, in fact, periodicity selective, as opposed to pitch selective, was not explicitly tested (e.g., with inharmonic tones, whose pitch does not match stimulus envelope periodicity). Moreover, inspection of the one published plot of cell discharge rate as a function of both pure-tone $f$ and harmonic-tone missing F0 (i.e., the isointensity function in FIGURE 3a, p. 1163) finds that the neuron increased its discharge rate two or more standard deviations above spontaneous rate for most of the missing F0s tested-that is, the cell was non-selectively sensitive to harmonic-tone stimulation. By contrast,

FIGURE 9. Spike dot rasters and F0 vs. spike-count poststimulus time histograms showing responses of a frequency-selective neuron [best frequency $(\mathrm{BF})=173 \mathrm{~Hz}$ ] to pure tones (left column), harmonic tones with energy at F0 (middle column), and harmonic tones missing F0 (B, right column) presented in quasi-free-field at $60 \mathrm{~dB}$ SPL (top row), $50 \mathrm{~dB}$ SPL (middle row), and $40 \mathrm{~dB}$ SPL (bottom row). This "F0 neuron" shows on-excitation during stimulation with pure tones and with harmonic tones when F0 is near BF (A), but there is no increase in firing during stimulation with harmonic tones missing $\mathrm{F} 0$, even when the missing F0 is near BF (B). (Reprinted by permission of the American Physiological Society. ${ }^{101}$ ) 


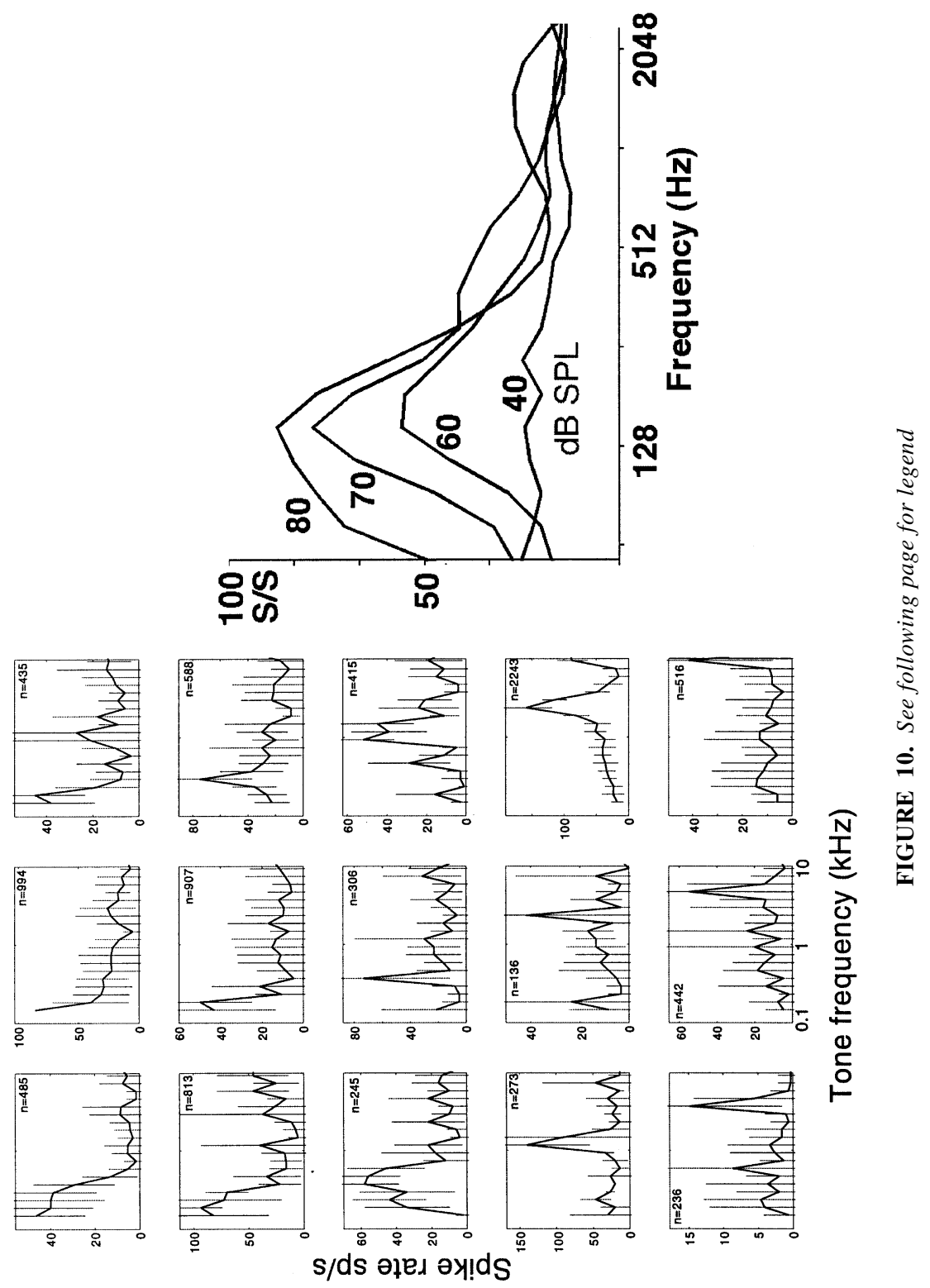


the neuron responded to a much more restricted range of pure-tone frequencies. In addition, the peak in the rate versus missing-F0 isointensity function is much broader than psychophysical thresholds for missing-F0 discrimination predict.

It may be the case that this particular neuronal population has a special role to play in pitch perception, but there are reasons to doubt that the central representation for pitch is based per se on a profile of firing rates among these units, that is, on which of these detectors are firing the most frequently at any given instant or over a window of time between a few milliseconds to hundreds of milliseconds. One problem is that many periodicity-tuned units found by Bendor and Wang, like many neurons in $\mathrm{A} 1$, had nonmonotonic rate-level functions. These units tend to respond best to tones with the right periodicities that are presented at moderate levels, not far above their response thresholds (it should be noted that the periodicity tuning of these units was only demonstrated for low sound pressure levels, not high ones). They respond with lower firing rates when the same tones are presented at higher, sound pressure levels (e.g., > 60-80 dB SPL). In contrast, perception of musical pitch (low-frequency harmonic complex tones), does not break down at higher levels (e.g., for music listening in concert halls and clubs) and is almost completely invariant over the whole dynamic range of hearing. The prevalence of nonmonotonic ratelevel functions means that different sets of these tuned periodicity units will respond maximally to the same tone presented at different sound levels. So, although such units may be able to give an economical explanation for why spectrally diverse stimuli presented at comparable levels have similar pitches, by the same argument, the same tones presented at different sound levels should have different pitches since different sets of units are activated. Although very slight shifts in pitch can be heard if levels are changed by $40 \mathrm{~dB}$ or more (maximally on the order of a few percent for pure tones and much less for complex tones), it is the extreme invariance of pitch with level that is striking. This invariance makes it possible for listeners to accurately match and discriminate pitches of tones even when their levels are randomly roved by tens of decibels.

The same problems arise when one attempts to account for the cortical representation of the pitches of low-frequency pure tones. In our single- and multiunit recordings of responses to pure tones in the core area of the alert macaques, ${ }^{18}$ we found a variety of response types. For tones presented at moderate levels (70-80 dB SPL), we observed ON responders that responded to their range of preferred tone frequencies during the presentation of sustained pure tones, OFF responders that responded immediately after the tone ceased, and complex responders that gave $\mathrm{ON}$ responses to one set of frequencies and OFF responses to another. Even for ON responders that had one preferred range of tone frequencies (FIG. 10), frequency tuning was relative-

FIGURE 10. Frequency selectivity in the core area of alert macaques is intensity dependent. (Left) Mean spike rate vs. pure-tone frequency plots measured at 15 extracellular recording sites ( 7 from single neurons, 8 from clusters of 2-3 neurons). These isointensity functions analyze frequency selectivity during pure-tone stimulation ( $\mathrm{ON}$ responses) at a fixed, moderate intensity (between 70-80 dB SPL near the tympanic membrane in the external auditory canal). Vertical bars in each plot show \pm 1 standard deviations from the mean spike rate computed across 10-20 repetitions of each frequency. (Right) Isointensity functions measured at four different tone intensities (40, 60, 70, $80 \mathrm{~dB}$ SPL) for a single neuron with a monotonic rate-level function. Note the increase in response bandwidth as tone intensity increases. 
ly broad (typically 1-2 octaves), level dependent, and subject to a high degree of response variability (FIG. 11). These properties appear to confound simple representations of tone frequency that are based on profiles of average firing rates across neuronal populations.

In their systematic single-unit studies of the representation of pure-tone frequency in anesthetized cat auditory cortex, Phillips et al. ${ }^{53}$ a half-decade earlier had found widespread nonmonotonic rate-level response functions and were forced to confront the problem of how such units could subserve a coherent rate-based representation of frequency (perceptually, pure-tone pitch). Their solution was to posit that the representation itself was level dependent, that is, a joint representation of

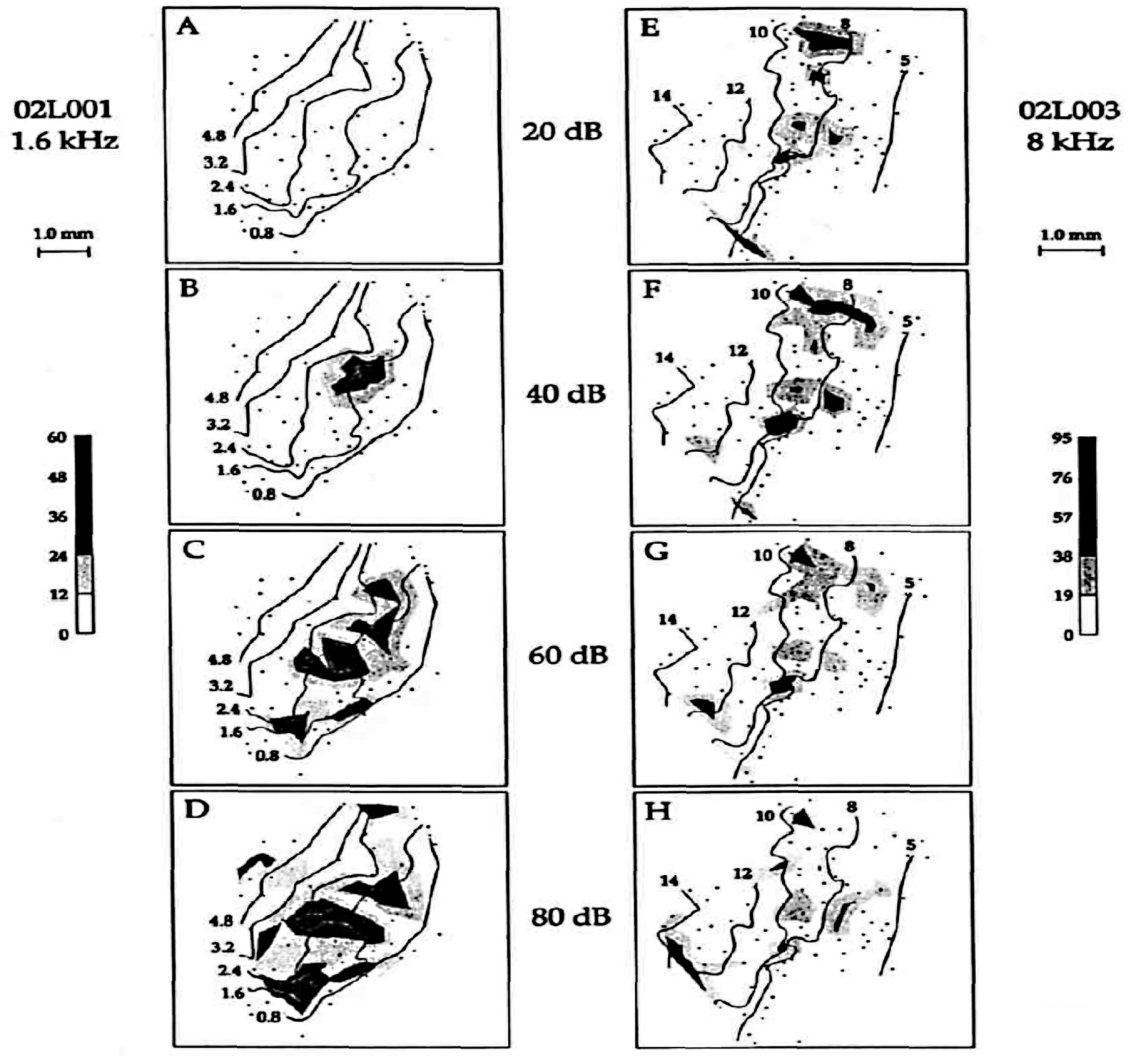

FIGURE 11. The topographic organization of rate-based neural representations of puretone frequency in A1 of anesthetized cats changes dramatically with tone intensity. (A-H) Flat maps of A1 (mostly its Layer IV) from two cats published by Phillips et al. ${ }^{53}$ Lines demarcate isofrequency contours, i.e., longitudinal patches of neurons that are maximally sensitive to the same frequency near their response threshold. Darker areas on each map indicate penetration sites where single-unit discharge rates evoked by best-frequency tones were highest. For example, in $\mathbf{B}$, stimulation with a best-frequency tone $(1.6 \mathrm{kHz})$ at $\leq 20 \mathrm{~dB}$ above response threshold (A) caused neurons in a discrete $1 \mathrm{~mm} \times 0.5 \mathrm{~mm}$ patch of A1 to fire. However, at higher intensities ( $\mathbf{C}$ and $\mathbf{D}$ ), the location of neurons maximally excited by the same tone frequency is markedly different. (Reprinted by permission of Springer, New York. ${ }^{53}$ ) 
both level and frequency. Unfortunately this solution simply intensifies the problem-now one must explain how level-invariant equivalence classes arise. Although connectionist learning models have been proposed that illustrate the general mechanisms by which pitch equivalence classes might arise through experience, such models have yet to show how robust equivalence classes could be formed using more realistic neuronal elements with highly variable responses coupled with nonmonotonic, level-dependent tuning.

In addition to meeting criteria related to perceptual acuity and constancy, viable neural codes for pitch must account for the functional dissociability of pitch change detection and pitch direction discrimination revealed by human lesion experiments. ${ }^{30,35}$ The existence of $\mathrm{A} 1$ and belt neurons that increase their firing rate more when the second tone is higher than the first tone than when it is lower, and vice versa, has been firmly established by Brosch and colleagues in three anesthetized Macaca fascicularis monkeys. ${ }^{108}$ To our knowledge, this remains the only systematic investigation of single- and multiunit responses to sequences of two pure tones in Old World monkeys with $\Delta \mathrm{fs}$ and ISIs similar to those used in psychoacoustic experiments. The prevalence of direction-sensitive units in different subdivisions of auditory cortex and their distribution in the left and right auditory cortices remain unknown.

Thus, an enigma persists concerning the nature of the neural codes and computations that support these universal and highly reliable perceptual invariances and equivalence classes at the cortical level. Whatever the code, it must behave in a highly reliable way under a huge variety of environmental conditions and for stimuli that are almost never present in the natural world. For the various reasons outlined here, we believe that the cortical representation of pitch is not likely to be based on either a simple temporal or rate-based code; nor is it likely to be based on idiosyncratic, special purpose assemblages of neural elements and interconnections. The neural representations and computations that subserve pitch perception may involve elegant and powerful information-processing principles and mechanisms that still elude us.

\section{ACKNOWLEDGMENTS}

This work was supported by NIH DC03328, DC006353, and DC00117; the Institute for Music and Brain Science; and the National Organization for Hearing Research. We thank the Pierfranco and Luisa Mariani Foundation, Dr. Maria Majno, Dr. Stefan Koelsch, and Mr. Steven Bohall; and our patients, colleagues, and students for their inspiration and contributions.

[Competing interests: The authors declare that they have no competing financial interests.]

\section{REFERENCES}

1. DE BoER, E. 1976. On the "residue" and auditory pitch perception. In Auditory System (Handbook of Sensory Physiology). W.D. Keidel \& W.D. Neff, Eds.: 479-583. Springer-Verlag. Berlin.

2. LICKLIDER, J.C.R. 1951. A duplex theory of pitch perception. Experientia VII: 128-134.

3. Terhardt, E. 1974. Pitch, consonance, and harmony. J. Acoust. Soc. Am. 55: 1061-1069.

4. Plomp, R. 1976. Aspects of Tone Sensation. Academic Press. London. p. 167.

5. Moore, B.C.J. 2003. An Introduction to the Psychology of Hearing. Academic Press. San Diego. 
6. Rameau, J.P. 1722. Treatise on Harmony. Dover (1971 reprint). New York.

7. Piston, W. \& M. DeVoto. 1987. Harmony, 5th Ed. Norton. New York.

8. Tomlinson, R.W.W. \& D.W.F. Schwartz. 1988 Perception of the missing fundamental in nonhuman primates. J. Acoust. Soc. Am. 84: 560-565.

9. WRIGHT, B.A. et al. 2000. Music perception and octave generalization in rhesus monkeys. J. Exp. Psychol. Gen. 129: 291-307.

10. IzUMI, A. 2000. Japanese monkeys perceive sensory consonance of chords. J. Acoust. Soc. Am. 108: 3073-3078.

11. Heffner, H. \& I.C. Whitfield. 1976. Perception of the missing fundamental by cats. J. Acoust. Soc. Am. 59: 915-919.

12. CYNX, J. 1986. Periodicity pitch in a species of songbird, the European starling (Sturnus vulgaris). Assoc. Res. Otolaryngol. Abstr. p. 138.

13. CapriancA, R.R., G.J. Rose \& E.A. BRenowitz. 1985. Time resolution in the auditory systems of anurans. In Time Resolution in Auditory Systems, A. Michelsen, Ed.: 2857. Springer-Verlag. Berlin.

14. FAY, R.R. 1985. Time processing by the auditory system of fishes. In Time Resolution in Auditory Systems. A. Michelsen, Ed.: 28-57. Springer-Verlag. Berlin.

15. BorCHGREVINK, H.M. 1975. Musical chord preferences in humans as demonstrated through animal experiments. Tideskrit for den Norske Laegeforening (Norwegian) 95: $356-358$.

16. Hulse, H.S., D.J. Bernard \& R.F. BraAten. 1995. Auditory discrimination of chordbased spectral structures by European starlings. J. Exp. Psychol. 124: 409-423.

17. JONES, E.G. et al. 1995. Subdivisions of macaque monkey auditory cortex revealed by calcium binding protein immunoreactivity. J. Comp. Neurol. 362: 1-19.

18. Tramo, M.J. 1998. Neural representations of acoustic information in relation to music and voice perception. Harvard University, Cambridge, MA. Ph.D. Dissertation.

19. Hackett, T.A., T.M. Preuss \& J.H. KaAs. 2001. Architectonic identification of the core region in auditory cortex of macaques, chimpanzees, and humans. J. Comp. Neurol. 441: 197-222.

20. Merzenich, M.M. \& J.F. BRugGe. 1973. Representation of the cochlear partition on the superior temporal plane of the macaque monkey. Brain Res. 50: 275-296.

21. PANDYA, D.N. 1995. Anatomy of the auditory cortex. Rev. Neurol. 151: 486-494.

22. KaAs, J.H., T.A. HacketT \& M.J. Tramo. 1999. Auditory processing in primate cerebral cortex. Curr. Opin. Neurobiol. 9: 164-170.

23. Rauschecker, J.F., B. Tian \& M.D. Hauser. 1995. Processing of complex sounds in the macaque nonprimary auditory cortex. Science 268: 111-114.

24. NefF, W.D., I.T. Diamond \& J.H. CASSEDAY. 1975. Behavioral studies of auditory discrimination: central nervous system. In Handbook of Sensory Physiology. W.D. Keidel \& W.D. Neff, Eds.: 307-400. Springer-Verlag. Berlin.

25. Thompson, R.F. 1960. Function of auditory cortex of cat in frequency discrimination. J. Neurophysiol. 23: 321-334.

26. Elliot, D.N. \& C. Trahoitis. 1972. Cortical lesions and auditory discrimination. Psychol. Bull. 77: 198-222.

27. Evarts, E.V. 1952. Effect of auditory cortex ablation on frequency discrimination in monkey. J. Neurophysiol. 15: 443-448.

28. Jerger, J. et al. 1969. Bilateral lesions of the temporal lobe: a case study. Acta Otolaryngol. Suppl. 252: $1-51$.

29. Jerger, J., L. LOVERING \& M. Wertz. 1972. Auditory disorder following bilateral temporal lobe insult: report of a case. J. Speach Hear Disord. 37: 523-535.

30. Tramo, M.J., G.D. Shah \& L.D. Braida. 2002. The functional role of auditory cortex in frequency processing and pitch perception. J. Neurophysiol. 87: 122-139.

31. Seashore, C.E., D. Lewis \& J.C. Saetvit. 1960. Seashore Measures of Musical Talents Manual. The Psychological Corporation. New York.

32. Tramo, M.J. 1990. Impaired perception of relative pure-tone pitch following bilateral lesions of auditory cortex in man. Soc. Neurosci. Abstr. 16: 580.

33. Tramo, M.J., A. Grant \& L.D. Braida. 1994. Psychophysical measurements of frequency difference limens for relative pitch discrimination reveal a deficit following bilateral lesions of auditory cortex. Soc. Neurosci. Abstr. 20: 325. 
34. KAZUI, S. et al. 1990. Subcortical auditory agnosia. Brain Lang. 38: 476-487.

35. Johnsrude, I.S., V.B. Penhune \& R.J. Zatorre. 2000. Functional specificity in the right human auditory cortex for perceiving pitch direction. Brain 123: 155-163.

36. DivenYI, P.L. \& A.J. Robinson. 1989. Nonlinguistic auditory capabilities in aphasia. Brain Lang. 37: 290-326.

37. Tramo, M.J., C.K. Koh, G.D. Shah \& L.D. Braida. 2004 Functional role of auditory cortex in virtual pitch perception. Soc. Neurosci. Abstr. 30 apu.sfn.org/content/Publications/AnnualMeeting/.

38. ZatorRe, R.J. 1988. Pitch perception of complex tones and human temporal-lobe function. J. Acoust. Soc. Am. 84: 566-572.

39. Robin, D., D. Tranel \& H. Damasio. 1990. Auditory perception of temporal and spectral events in patients with focal left and right cerebral lesions. Brain Lang. 39: 539555.

40. Massopust, L.C. JR., et al. 1967. Changes in auditory frequency discrimination thresholds after temporal cortex ablations. Exp. Neurol. 19: 245-255.

41. Harrington, D.L., R.S. HeFFner \& H.E. HeFFner. 2001. An investigation of sensory deficits underlying the aphasia-like behavior of macaques with auditory cortex lesions. Neuroreport 12: 1217-1221.

42. Sidtis, J.J. \& B.T. VolPE. 1988. Selective loss of complex-pitch or speech discrimination after unilateral lesion. Brain Lang. 34: 235-245.

43. Bharucha, J.J., M.J. Tramo \& R.J. Zatorre. 1993. Abstraction of the missing fundamental following bilateral lesions of auditory cortex. Soc. Neurosci. Abstr. 19: p. 1687.

44. Pantev, C. et al. 1996. Binaural fusion and the representation of virtual pitch in the human auditory cortex. Hear. Res. 100: 164-170.

45. Langner, G. et al. 1997. Frequency and periodicity are represented in orthogonal maps in the human auditory cortex: evidence from magnetoencephalography. J. Comp. Physiol. 181: 665-676.

46. ZatorRe, R. et al. 1992. Lateralization of phonetic and pitch discrimination in speech processing. Science 256: 846-849.

47. Tramo, M.J. et al. 2001. Neurobiological foundations for the theory of harmony in Western tonal music. Ann. N. Y. Acad. Sci. 930: 92-116.

48. GAAB, N., C. GASER, T. ZAehle, et al. 2003. Functional anatomy of pitch memory-a fMRI study with sparse temporal sampling. Neuroimage 19: 1417-1426.

49. Patterson, R.D. et al. 2002. The processing of temporal pitch and melody information in auditory cortex. Neuron 36: 767-76.

50. Penagos, H., J.R. Melcher \& A.J. Oxenham. 2004. A neural representation of pitch salience in nonprimary human auditory cortex revealed with functional magnetic resonance imaging. J. Neurosci. 24: 6810-6815.

51. Moore, B.C.J., R.W. Peters \& B.R. Glasberg. 1985. Thresholds for the detection of inharmonicity in complex tones. J. Acoust. Soc. Am. 77: 1985.

52. Wier, C.C., W. JeSteadT \& D.M. GreEn. 1977. Frequency discrimination as a function of frequency and sensation level. J. Acoust. Soc. Am. 61: 178-184.

53. PhiLLIPS, D.P. et al. 1994. Level-dependent representation of stimulus frequency in cat primary auditory cortex. Exp. Brain Res. 102: 210-226.

54. Cohen, M.A., S. Grossberg \& L.L. Wyse. 1994. A spectral network model of pitch perception. J. Acoust. Soc. Am. 98: 862-879.

55. BHARUCHA, J.J. 1991. Pitch, harmony and neural nets: a psychological perspective. In Connectionism and Music. P. Todd \& G. Loy, Eds.: 84-99. MIT Press. Cambridge.

56. SutTer, M.L. \& C.E. SChreinER. 1991. Physiology and topography of neurons with multipeaked tuning curves in cat primary auditory cortex. J. Neurophysiol. 65: 1207-1226.

57. FitZPATRICK, D.C. et al. 1993. Combination-sensitive neurons in the primary auditory cortex of the mustached bat. J. Neurosci. 13: 931-940.

58. SugA, N. \& W.E. O'NeIL. 1979. Harmonic-sensitive neurons in the auditory cortex of the Mustache bat. Science 203: 270-274.

59. DEAR, S.P. et al. 1993. Tonotopic and functional organization in the auditory cortex of the big brown bat, Eptesicus fuscus. J. Neurophysiol. 70: 1988-2009. 
60. Moore, B.C.J. 1997. An Introduction to the Psychology of Hearing, 4th Ed. Academic Press. London. p. 373.

61. Meddis, R. \& L. O’MARD. 1997 A unitary model of pitch perception. J. Acoust. Soc. Am. 102: 1811-1820.

62. Cariani, P.A. \& B. Delgutte. 1996. Neural correlates of the pitch of complex tones. I. Pitch and pitch salience. II. Pitch shift, pitch ambiguity, phase-invariance, pitch circularity, and the dominance region for pitch. J. Neurophysiol. 76: 1698-1734.

63. CARIANI, P. 1999. Temporal coding of periodicity pitch in the auditory system: an overview. Neural Plast. 6: 147-172.

64. Palmer, A.R. 1992. Segregation of the responses to paired vowels in the auditory nerve of the guinea pig using autocorrelation. In The Auditory Processing of Speech S.M.E.H., Ed.: 115-124. Mouton de Gruyter. Berlin.

65. Hirahara, T., P. Cariani \& B. Delgutte. 1996. Representation of low-frequency vowel formants in the auditory nerve. In Proceedings, ESCA Research Workshop on The Auditory Basis of Speech Perception, Keele University, United Kingdom. July $15-19,1996$.

66. Goldstein, J.L. \& P. SRulovicz. 1977. Auditory-nerve spike intervals as an adequate basis for aural frequency measurement. In Psychophysics and Physiology of Hearing. E.F. Evans \& J.P. Wilson, Eds. Academic Press. London.

67. Langner, G. 1992. Periodicity coding in the auditory system. Hear. Res. 60: 115-142.

68. LANGner, G. \& C.E. SchreINER. 1988. Periodicity coding in the inferior colliculus of the cat. I. Neuronal Mechanisms. J. Neurophysiol. 60: 1799-1822.

69. Rose, G.J. \& R.R. CAPRIANICA. 1985. Sensitivity to amplitude modulated sounds in the anuran auditory nervous system. J. Neurophysiol. 53: 446-465.

70. Epping, W.J.M. \& J.J. EgGermont. 1986. Sensitivity of neurons in the auditory midbrain of the grassfrog to temporal characteristics of sound. I Stimulation with acoustic clicks. Hear. Res. 24: 37-54.

71. Epping, W.J.M. \& J.J. EgGermont. 1986. Sensitivity of neurons in the auditory midbrain of the grassfrog to temporal characteristics of sound. II. Stimulation with amplitude modulated sounds. Hear. Res. 24: 55-72.

72. Rees, A. \& A.R. Møller. 1983. Responses of neurons in the inferior colliculus of the rat to AM and FM tones. Hear. Res. 10: 301-330.

73. KRISHnA, M.B.S. \& M.N. SEMPLE. 2000. Auditory temporal processing: responses to sinusoidally amplitude-modulated tones in inferior colliculus. J. Neurophysiol. 84: 255-273.

74. DE BOER, E. 1956. On the "residue" in hearing. University of Amsterdam.

75. SChreiner, C.E. \& G. LANGNER. 1988. Coding of temporal patterns in the central auditory system. In Auditory Function: Neurobiological Bases of Hearing. G.M. Edelman, W.E. Gall \& W.M. Cowan, Eds.: 337-362. Wiley. New York.

76. RHODE, W.S. 1995. Interspike intervals as correlates of periodicity pitch in cat cochlear nucleus. J. Acoust. Soc. Am. 97: 2414-2429.

77. Rhode, W.S. \& S. Greenberg. 1992. Physiology of the cochlear nuclei. In The Mammalian Auditory Pathway: Neurophysiology. A.N. Popper \& R.R. Fay, Eds.: 94-152. Springer-Verlag. New York.

78. YounG, E.D. 1984. Response characteristics of neurons in the cochlear nuclei. In Hearing Science. C. Berlin, Ed.: 423-460. College Hill. San Diego.

79. Colburn, S. \& N.I. Durlach. 1978. Models of binaural interaction. In Handbook of Perception. E.C. Carterette \& M.P. Friedman, Eds.: 467-518. Academic Press. New York.

80. Depireux, D.A. \& S.A. Shamma. 1998. Neuronal correlates of pitch in the inferior colliculus. Assoc. Res. Otolaryngol. Abstr. 20: 182.

81. GREenberg, S. 1980. Neural temporal coding of pitch and vowel quality: human frequency, following response studies of complex signals. UCLA Working Papers in Phonetics \#52, Los Angeles.

82. DE RibAuPIERRE, F. 1997. Acoustical information processing in the auditory thalamus and cerebral cortex. In The Central Auditory System. G. Ehret \& R. Romand, Eds.: 317-397. Oxford University Press. New York.

83. Makela, J.P. et al. 1990. Steady-state responses from the cat auditory cortex. Hear. Res. 45: 41-50. 
84. Goldstein, M.H., N.Y.-S. Kiang \& R.M. BRown. 1959. Responses of auditory cortex to repetitive acoustic stimuli. J. Acoust. Soc. Am. 31: 356-364.

85. GoldSTEIN, M.H., JR. \& N.Y.S. KIANG. 1958. Synchrony of neural activity in electric responses evoked by transient acoustic stimuli. J. Acoust. Soc. Am. 30: 107-114.

86. Steinschneider, M., J. Arezzo \& H.G. Vaughan Jr. 1980. Phase locked cortical responses to a human speech sound and low frequency tones in the monkey. Brain Res. 198: 75-84.

87. STEINSCHNEIDER, M. et al. 1998. Click train encoding in primary auditory cortex of the awake monkey: evidence for two mechanisms subserving pitch perception. J. Acoust. Soc. Am. 104: 2395-2955.

88. De Ribaupierre, F., M.H. Goldstein JR. \& G. Yeni-Komshian. 1972. Cortical coding of repetitive acoustic pulses. Brain Res. 48: 205-225.

89. Chow, K.L. 1951. Numerical estimates of the auditory central nervous system of the rhesus monkey. J. Comp. Neurol. 95: 159-175.

90. VILLA, A.E.. 2000. Empirical evidence about temporal structure in multi-unit recordings. In Time and the Brain. R. Miller, Ed.: 1-52. Harwood Academic Publishers. Australia.

91. Eggermont, J.J. 1990. The Correlative Brain: Theory and Experiment in Neural Interaction. Springer-Verlag. Berlin. p. 307.

92. EgGermont, J.J. \& G. SMith. 1995. Synchrony between single-unit activity and local field potentials in relation to periodicity coding in primary auditory cortex. J. Neurophysiol. 73: 227-245.

93. EgGermont, J.J. 1993. Functional aspects of synchrony and correlation in the auditory nervous system. Concep. Neurosci. 4: 105-129.

94. SingER, W. 1994. Time as coding space in neocortical processing. In Temporal Coding in the Brain. G. Buzsáki et al., Eds.: 51-80. Springer-Verlag. Berlin.

95. Espinoza, I.E. \& G.L. Gerstein. 1988. Cortical auditory neuron interactions during presentation of 3-tone sequences: effective connectivity. Brain Res. 450: 39-50.

96. EMmers, R. 1981. Pain: A Spike-Interval Coded Message in the Brain. Raven Press. New York.

97. MountCastle, V. 1993. Temporal order determinants in a somatosthetic frequency discrimination: sequential order coding. Ann. N. Y. Acad. Sci. 682: 151-170.

98. Abeles, M. et al. 1993. Spatiotemporal firing patterns in the frontal cortex of behaving monkeys. J. Neurophysiol. 70: 1629-1638.

99. Lestienne, R. \& B.L. StrehleR. 1987. Time structure and stimulus dependence of precise replicating patterns present in monkey cortical neuronal spike trains. Brain Res. 43: 214-238.

100. DeCharms, R.C. \& M.M. Merzenich. 1996. Primary cortical representation of sounds by the coordination of action-potential timing. Nature 381: 610-613.

101. Schwarz, D.W.F. \& R.W.W. TomLinson. 1990. Spectral response patterns of auditory cortical neurons to harmonic complex tones in alert monkey (Macaca mulatta). J. Neurophysiol. 64: 282-298.

102. Riquimaroux, H. \& T. Hashikawa. 1994. Units in the primary auditory cortex of the Japanese monkey can demonstrate a conversion of temporal and place pitch in the central auditory system. Journal de Physique IV 4: 419-425.

103. BENDOR, D. \& X. WANG. 2005. The neuronal representation of pitch in primate auditory cortex. Nature 436: 1161-1165.

104. American National Standards Institute (ANSI). 1960, 1994. American National Standard Acoustical Terminology for Physiological and Psychological Acoustics. ANSI Suppl 1.1. American National Standards Institute. New York.

105. Tramo, M.J., J.J. Bharucha \& F.E. Musiek. 1990. Music perception and cognition following bilateral lesions of auditory cortex. J. Cog. Neurosci. 2: 195-212.

106. WARRIER, C.M. \& R.J. ZATORRE. 2004. Right temporal cortex is critical for utilization of melodic contextual cues in a pitch constancy task. Brain 127: 1616-1625.

107. Tramo, M.J. 2001. Music of the hemispheres. Science 291: 54-56.

108. Brosch, M., A. Schulz \& H. Scheich. 1999. Processing of sound sequences in macaque auditory cortex:response enhancement. J. Neurophysiol. 82: 1542-1559. 